\title{
THz GENERATION BY OPTICAL RECTIFICATION FOR A NOVEL SHOT TO SHOT SYNCHRONIZATION SYSTEM BETWEEN ELECTRON BUNCHES AND FEMTOSECOND LASER PULSES IN A PLASMA WAKEFIELD ACCELERATOR*
}

\author{
H. Schlarb, DESY, Hamburg, Germany
}

crystal $\left(\mathrm{LiNbO}_{3}\right)(\mathrm{PPLN})$. These pulses allow to energy modulate the electron bunches shot to shot before the plasma in order to achieve the time resolution [3].

This paper focuses on the generation of $\mathrm{THz}$ pulses. We present our investigation of the influence of the optical properties as well as the theoretical description of the $\mathrm{THz}$ generation on the conversion efficiency as well on the optimum crystal length for the generation length of short $\mathrm{THz}$ pulses.

The paper is organized as follows. First, we derive the general equations for the description of the $\mathrm{THz}$ generation and then we introduce two different methods in order to include the effects of the laser pump on the crystal. Therefore we compare the corresponding results for the efficiency as well as for optimum crystal length of the $\mathrm{THz}$ generation. The conclusions finalize this work.

\section{MODELING THE THz GENERATION}

Starting from the Maxwell equations the following one dimensional system of coupled differential equations for the laser pulse $E_{\mathrm{L}}\left(\omega_{\mathrm{L}}, z\right)=A_{\mathrm{L}}\left(\omega_{\mathrm{L}}\right) \mathrm{e}^{-l k\left(\omega_{\mathrm{L}}\right) z}$ and for the $\mathrm{THz}$ wave $E_{\mathrm{T}}\left(\omega_{\mathrm{T}}, z\right)=A_{\mathrm{T}}\left(\omega_{\mathrm{T}}\right) \mathrm{e}^{-l k\left(\omega_{\mathrm{T}}\right) z}$

$$
\begin{aligned}
& \left(\frac{\partial^{2}}{\partial z^{2}}+\frac{\omega_{\mathrm{T}}^{2}}{c^{2}} \varepsilon\left(\omega_{\mathrm{T}}\right)\right) E_{\mathrm{T}}\left(\omega_{\mathrm{T}}, z\right)=G_{\mathrm{T}}\left(\omega_{\mathrm{T}}, \omega_{\mathrm{L}}, z\right) \\
& \left(\frac{\partial^{2}}{\partial z^{2}}+\frac{\omega_{\mathrm{L}}^{2}}{c^{2}} \varepsilon\left(\omega_{\mathrm{L}}\right)\right) E_{\mathrm{L}}\left(\omega_{\mathrm{L}}, z\right)=G_{\mathrm{L}}\left(\omega_{\mathrm{L}}, \omega_{\mathrm{T}}, z\right),
\end{aligned}
$$

can be derived [4-7]. Here $\varepsilon(\omega)$ denotes the generalized (complex) dielectric function and the inhomogeneous term $G_{\mathrm{L}}$ and $G_{\mathrm{T}}$ are related to the nonlinear polarizations in the optical and $\mathrm{THz}$ frequency range respectively.

A simplification for this system is the slope varying approximation (SVA), where the second spatial derivatives of the amplitudes $A_{\mathrm{m}}$ with $\mathrm{m} \in \mathrm{L}, \mathrm{T}$ are neglected. This approach is used in almost all investigations $[4,8-10]$ and leads to coupled system of linear differential of the first order,

$$
\left(\frac{\partial}{\partial z}+\frac{\alpha_{\mathrm{m}}\left(\omega_{\mathrm{m}}\right)}{2}\right) A_{\mathrm{m}}\left(\omega_{\mathrm{m}}, z\right)=G_{\mathrm{m}}^{\mathrm{SVA}}\left(\omega_{\mathrm{T}}, \omega_{\mathrm{L}}, z\right)
$$

where $\alpha_{\mathrm{m}}$ indicates the adsorption coefficient in the optical $(\mathrm{m}=\mathrm{L})$ and in the $\mathrm{THz}$ range $(\mathrm{m}=\mathrm{T})$ respectively and the inhomogeneous terms become [11]

$$
G_{\mathrm{m}}^{\mathrm{SVA}}=\imath G_{\mathrm{m}} \frac{\mathrm{e}^{\imath k\left(\omega_{\mathrm{T}}\right) z}}{2 k\left(\omega_{\mathrm{m}}\right)} .
$$

The assumption $G_{\mathrm{L}}=0$ leads to the decoupling of the system and only Eq. (1) or Eq. (3) with $\mathrm{m}=\mathrm{T}$ has to be 
solved. In this way the direct depletion of the laser pump is neglected. Nevertheless, in this approximation used in several works [3,7-10], the inhomogeneous term of Eq. (1) can be written as $G_{\mathrm{T}}=-\mu_{0} \omega_{\mathrm{T}}^{2} P_{\mathrm{NL}}\left(\omega_{\mathrm{T}}, z\right)$, where the nonlinear polarization is given by

$$
P_{\mathrm{NL}}\left(\omega_{\mathrm{T}}, z\right)=\varepsilon_{0} \chi^{(2)}(z) \int_{0}^{\infty} \mathrm{d} \omega E_{\mathrm{L}}\left(\omega_{\mathrm{T}}+\omega, z\right) E_{\mathrm{L}}^{*}(\omega, z) .
$$

Here $\varepsilon_{0}$ and $\chi^{(2)}(z)$ indicate the vacuum dielectric constant and the second order nonlinear susceptibility respectively.

The optical properties of the material are encoded in the complex dielectric function $\varepsilon(\omega)$, which is related to the wave vector $k(\omega)$, the refractive index $n(\omega)$ and the adsorption coefficient $\alpha(\omega$ by

$$
n(\omega)=\frac{k(\omega) c}{\omega}=\Re \sqrt{\varepsilon(\omega)} \quad \alpha(\omega)=\frac{2 \omega}{c} \mathfrak{I} \sqrt{\varepsilon(\omega)} .
$$

For the polarization only the knowledge of the wave vector in the frequency region around laser central frequency $\omega_{0}$ is needed. For this purpose, we describe the refractive index squared as function of the frequency using the Sellmeier equation given in [12]. Nevertheless, performing a Taylor expansion of the wave vector for the term $k\left(\omega_{\mathrm{T}}+\omega\right)-k(\omega)$, which enters in the polarization integral, a linear approximation,

$$
k\left(\omega_{\mathrm{T}}+\omega_{\mathrm{L}}\right)-k\left(\omega_{\mathrm{L}}\right) \approx \frac{n_{\mathrm{opt}}^{\mathrm{gr}}}{c} \omega_{\mathrm{T}}
$$

can be used [8-10]. In this manuscript we will utilize and compare both descriptions in order to evaluate the effects of the whole dispersion relation.

For the THz region we use a physical motivated description for $\varepsilon\left(\omega_{\mathrm{T}}\right)$ based on the oscillator model given in [13].

\section{INFLUENCE OF THE FREE CARRIERS}

An important effect is given by the free carries (FC), which are generated by the pump adsorption in the material $[5,9$, $10,14]$ and lead to a decreasing of the pump intensity in the crystal. In this manuscript we follow two different strategies in order to systematically consider the influence of the FC.

\section{(a) Modification of the Dielectric Function}

In this first approach (a) we decoupling the differential equations by setting $G_{L}=0$ and we systematically modify the dielectric function, which encodes the FC contributions to the optical properties as following $[5,11,14,15]$

$$
\varepsilon_{\mathrm{tot}}\left(\omega_{\mathrm{T}}\right)=\varepsilon_{\mathrm{osc}}\left(\omega_{\mathrm{T}}\right)-\frac{\omega_{\mathrm{pl}}^{2}}{\omega_{\mathrm{T}}^{2}+i \omega_{\mathrm{T}} / \tau_{\mathrm{sc}}},
$$

The contribution of the free carries encoded the second term of $\varepsilon_{\text {tot }}\left(\omega_{\mathrm{T}}\right)$ are implemented along the line of a Drude model with electron scattering length $\tau_{\mathrm{sc}}[10]$ and with the plasma frequency $\omega_{\mathrm{pl}}^{2}$ proportional to the density of free charge carries $\rho_{\mathrm{FC}}$. In our previous works $[5,11,14,15]$ we developed a phenomenological description for $\rho_{\mathrm{FC}}$ in a PPLN,

$$
\rho_{\mathrm{FC}}\left(F_{\mathrm{L}}\right)=\left\{\begin{aligned}
\rho_{3 \mathrm{PA}}\left(F_{\mathrm{L}}\right) & F_{\mathrm{L}} \leq F_{0} \\
\rho_{\mathrm{s}}-A \mathrm{e}^{-a\left(F_{\mathrm{L}}-F_{0}\right)} & F_{\mathrm{L}}>F_{0},
\end{aligned}\right.
$$

For fluences smaller than a transition fluence $F_{0}$ we describe the FC density by the three-photon-adsorption process (3PA) of the pump beam in the medium. For larger fluences we model in this way a saturation of the FC density in agreement with experimental observations [10].

\section{(b) Decreasing of the Pump Intensity}

As second approach (b) in order to include the effects of the free carries have to be considered directly the decreasing of the pump intensity [7] induced by the three photon adsorption and given in frequency domain by $[11,14,15]$

$$
I_{\mathrm{L}}\left(\omega_{\mathrm{T}}, z\right)=\mathrm{e}^{-l q z} \sum_{n=0}^{\infty} \tilde{I}_{n} z^{n} \text {, with } \tilde{I}_{n}=\frac{u_{n}}{2 \sigma_{n} \sqrt{\pi}} \mathrm{e}^{-\frac{\omega_{\mathrm{T}}^{2}}{4 \sigma_{n}^{2}}}
$$

where we use the following notation $u_{n}=$ $\left(\begin{array}{c}-1 / 2 \\ n\end{array}\right) I_{0}\left(2 \gamma_{3} I_{0}^{2}\right)^{n}, \sigma_{n}^{2}=\frac{2}{\tau^{2}}(2 n+1)$ and $q=\omega_{\mathrm{T}} n_{\mathrm{opt}}^{\mathrm{gr}} / c$.

The nonlinear polarization and the intensity are proportional, if $P_{\mathrm{T}}$ includes quadratic terms of the electrical field only. Therefore, within the linear approximation, see. Eq. (7), the inhomogeneous terms of the coupled system in SVA given by Eq. (3) read

$$
\begin{aligned}
& G_{\mathrm{T}}\left(\omega_{\mathrm{T}}, z\right)=-l \frac{\omega_{\mathrm{T}}^{2}}{2 k\left(\omega_{\mathrm{T}}\right) c^{3} n_{0} \varepsilon_{0}} I_{\mathrm{L}}\left(\omega_{\mathrm{T}}, z\right) \mathrm{e}^{i k\left(\omega_{\mathrm{T}}\right) z} \\
& \left.G_{\mathrm{L}} \omega_{\mathrm{L}}, z\right)=\mathrm{FT}_{\mathrm{t} \rightarrow[}\left[-\frac{\gamma_{3}}{4} n\left(\omega_{0} c \varepsilon_{0}\right)^{2} E_{\mathrm{L}}^{3}(t)\left(E_{\mathrm{L}}^{*}(t)\right)^{2}\right],
\end{aligned}
$$

where $\mathrm{FT}_{\mathrm{t} \rightarrow}$ indicate the Fourier transform from time to frequency domain. Here we neglect the linear adsorption in the optical regime and we use $\alpha_{\mathrm{T}}$ calculated from the oscillator model dielectric function given in [13].

For a Gaussian pulse an analytic expression for the amplitude $A_{\mathrm{T}}$ can be derived as $A_{\mathrm{T}}\left(\omega_{\mathrm{T}}, z\right)=\sum_{n=0}^{\infty} a_{n} z^{n}$, where the coefficient $a_{n}$ are given by $[14,15]$

$$
a_{n}=\left\{\begin{array}{ll}
\mathrm{e}^{-l q z} \sum_{m=1}^{\infty} \frac{p_{n+m}}{b} \frac{(n+m) !}{m !} & \text { if } n \geq 1 ; \\
\mathrm{e}^{-\alpha z / 2} \sum_{m=1}^{\infty} \frac{p_{n+m}}{b} \frac{(n+m) !}{m !} & \text { if } n=0 .
\end{array},\right.
$$

with $b=\alpha / 2-\imath \Delta k_{0}$ and $p_{m}=-l \frac{\Omega \chi_{\mathrm{eff}}^{(2)}}{2 k c^{3} n_{0} \varepsilon_{0}} \tilde{I}_{n}$.

\section{RESULTS}

In this manuscript we investigate a periodic polarized congruent lithium niobate crystal with $\chi^{(2)}(z)=$ $\chi_{\mathrm{eff}}^{(2)} \mathrm{e}^{-l 2 \pi z / \Lambda}$, where the parameter $\chi_{\mathrm{eff}}^{(2)}=336 \mathrm{pm} \mathrm{V}^{-1}$ is the effective second order nonlinear susceptibility and $\Lambda=237.74 \mu \mathrm{m}$ is the quasi-phase-matching orientationreversal period. We consider a Gaussian laser beam pulse with central wave length $\lambda_{0}=1030 \mathrm{~nm}$ and a pulse duration at full width of half-maximum $\tau_{\mathrm{FWHM}}=25 \mathrm{fs}[3-5,8,10$, $11,14,15]$.

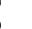
(n)

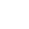

.

产

.




\section{Conversion Efficiency}

Because for the optimization of the synchronization an efficient and stable generation of THz pulses is a fundamental task, the conversion efficiency of the $\mathrm{THz}$ generation, defined as $[3,4]$

$$
\eta=\frac{\pi \epsilon_{0} c \int_{0}^{\infty} \mathrm{d} \omega_{\mathrm{T}} n\left(\omega_{\mathrm{T}}\right)\left|E_{\mathrm{T}}\left(\omega_{\mathrm{T}}, z\right)\right|^{2}}{F_{\mathrm{L}}},
$$

where $F_{\mathrm{L}}$ indicates the pump fluence, has to be maximized In order to understand the dependence of $\eta$ on the optical properties we calculate the efficiency by solving directly the equation of motion as differential equation of the second order, i.e. Eq. (1) as well as using the SVA, i.e. Eq. 3. In Fig. 1 we compare the results for the conversion efficiency $\eta$ for a fixed pump fluence $F_{\mathrm{L}}=5 \mathrm{~mJ} \mathrm{~cm} \mathrm{~cm}^{-2}$ as function of the crystal length $L$ between the second order calculation (solid lines) and the SVA (dashed lines). All results in this figure are obtained using the approach (a) for the description of FC, because at this fluence the results with the strategy (b) does not present significative deviations.

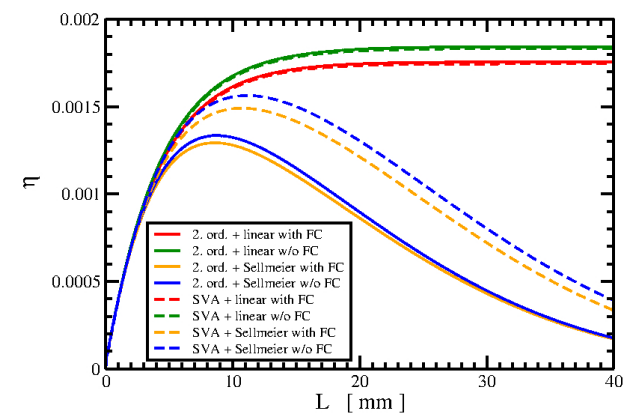

Figure 1: The conversion efficiency $\eta$ for a fixed pump fluence $F_{\mathrm{p}}=5 \mathrm{~mJ} \mathrm{~cm}^{-2}$ as function of the crystal length $L$ for the second order calculation (solid lines) and the SVA (dashed lines) labeled by different colors as indicated.

In the linear approximation is used, see. Eq. (7), we note a saturation of $\eta$ for large crystal lengths as well as deviations between the second order and SVA calculations.

Using the Sellmeier equation, the conversion efficiency $\checkmark$ shows a local maximum about around $L \approx 10 \mathrm{~mm}$ and is ह a decreasing function of the larger crystal length. We note effects of the FC contributions as well as larger deviations between the results coming between from second order cal$\overline{ \pm}$ culation and SVA. Therefore, at small fluences the second order dynamics effects are stronger than the contribution of the free charge carries. However, at large laser pump intensities this finding changes and the contribution of FC plays a key role, whereas the effects of the second order can be neglected $[5,15]$.

Therefore, for the investigation of the $\eta$ at higher pump fluence we can restrict ourselves to the SVA calculation and we consider the efficiency as function of $F_{\mathrm{L}}$ at fixed crystal length using both approaches for the inclusion of the FC contributions. In Fig. 2 we compare $\eta$ calculated obtained within a (minimal) depleted calculation (b) for a crystal length of

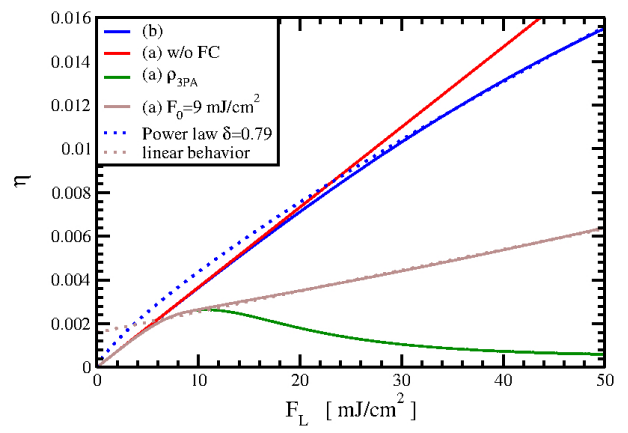

Figure 2: Comparison of $\eta$ obtained within a (minimal) depleted calculation (b) for a crystal length of $L=40 \mathrm{~mm}$ as function of $F_{\mathrm{L}} \eta$ calculated in SVA (undepleted) using the linear approximation within the approach (a) for the different parameterizations of the FC density as well as for vanishing FC density as indicated.

$L=40 \mathrm{~mm}$ as function of $F_{\mathrm{L}}$ as well as the efficiency $\eta$ calculated in SVA within the linear approximation within the approach (a) for the different parameterizations of the FC density. For comparison, the results for the efficiency calculated at vanishing FC are plotted, which shows a linear behavior.

In the approach (a), for unsaturated density of FC, the $\eta$ holds a non-monotonic behavior with a local maximum around $10 \mathrm{~mJ} \mathrm{~cm} \mathrm{~cm}^{-2}$ and then it decreases to an asymptotic value for large fluences, whereas for saturated $\rho_{\mathrm{FC}}$ with $F_{0}=9 \mathrm{~mJ} \mathrm{~cm}^{-2}$ exhibits a monotonic increasing behavior Nevertheless, a change of the slope of this behavior occurs at the value of the fluence, where the local maximum of the efficiency for the unsaturated FC density is located.

Using the strategy (b) the depleted calculation shows a deviation from the linear dependence on $F_{\mathrm{L}}$. Furthermore, the asymptotic behavior for large fluences follows a power law $\eta \propto F_{\mathrm{L}}^{\delta}$ with $\delta=0.79$, whereas the efficiency calculated including the FC contribution parameterized by $F_{0}=9 \mathrm{~mJ} \mathrm{~cm}^{-2}$ shows a linear asymptotic functional dependence.

\section{Optimum Crystal Length}

For the development of the planned feed-back system a estimation of the optimum crystal length $l_{0}$ for the generation is a fundamental task. For this purpose, we restrict ourselves on the SVA and we firstly consider the approach (a). Under these assumptions, the absolute value of the field squared can be factorized as $[5,7,15]$

$$
\left|A_{\mathrm{T}}\left(\omega_{\mathrm{T}}, z\right)\right|^{2}=\left|F\left(\omega_{\mathrm{T}}\right)\right|^{2}\left|L_{\mathrm{Gen}}\left(\omega_{\mathrm{T}}, z\right)\right|^{2},
$$

where we define

$$
L_{\mathrm{Gen}}\left(\omega_{\mathrm{T}}, z\right)=\int_{0}^{\infty} \mathrm{d} \omega E_{\mathrm{L}}\left(\omega_{\mathrm{T}}+\omega, z\right) E_{\mathrm{L}}^{*}(\omega, z) D\left(\omega, \omega_{\mathrm{T}}\right),
$$

with

$$
D\left(\omega, \omega_{\mathrm{T}}\right)=\frac{\epsilon_{0} \chi_{\mathrm{eff}}^{(2)}\left(\mathrm{e}^{-\imath \Delta k z}-\mathrm{e}^{\alpha_{\mathrm{T}} z / 2}\right)}{P_{\mathrm{NL}}\left(\omega_{\mathrm{T}}, z\right)} \frac{1}{\iota \Delta k-\alpha_{\mathrm{T}} / 2}
$$


with the mismatching $\Delta k=k\left(\omega_{\mathrm{T}}+\omega_{\mathrm{L}}\right)-k\left(\omega_{\mathrm{L}}\right)-k\left(\omega_{\mathrm{T}}\right)+$ $\frac{2 \pi}{\Lambda}$. The quantity has the unit of a length and can be considered as an effective generation length of the $\mathrm{THz}$ generation. It includes the whole spatial dependence of the electric field $A_{\mathrm{T}}$ and depends strongly on the optical properties of the material. We can generalize $\left|L_{\mathrm{Gen}}\right|^{2}$ for the approach (b) in the linear approximation as

$$
\left|L_{\mathrm{Gen}}\left(\omega_{\mathrm{T}}, z\right)\right|^{2}=\frac{\left|A_{\mathrm{T}}\left(\omega_{\mathrm{T}}, z\right)\right|^{2}}{\left|F_{(\mathrm{b})}\left(\omega_{\mathrm{T}}\right)\right|^{2}}
$$

with

$$
F_{(\mathrm{b})}\left(\omega_{\mathrm{T}}\right)=\omega_{\mathrm{T}}^{2} P_{\mathrm{NL}}\left(\omega_{\mathrm{T}}, z=0\right) /\left(2 k\left(\omega_{\mathrm{T}}\right) c^{2} \epsilon_{0}\right) .
$$

Therefore, at the optimum crystal length $l_{0}$ for the $\mathrm{THz}$ generation, the generation length, i.e. the quantity

$$
L_{\max }\left(\omega_{\mathrm{T}}\right)=\left|L_{\mathrm{Gen}}\left(\omega_{\mathrm{T}}, l_{0}\right)\right|,
$$

is a frequency dependent measure for the highest possible conversion efficiency, whereas $\eta$ is an inclusive measure of the efficiency over the full spectrum.

In general $L_{\max }\left(\omega_{\mathrm{T}}\right)$ and $l_{0}\left(\omega_{\mathrm{T}}\right)$ as functions of the frequency exhibit a maximum at the critical frequency $\omega_{\mathrm{cr}}$, where the mismatching vanishes, i.e. $\Delta k\left(\omega_{\mathrm{cr}}\right)=0$. According on the using of the linear approximation or the complete Sellmeier equation and on the choice for treatment of the influence of the free carries, i.e. approach (a) or (b), following features can be summarized:

i) In the approach (a) without the FC contribution the $L_{\max }\left(\omega_{\mathrm{T}}\right)$ is independent from the laser fluence.

ii) In the approach (a) with non-vanishing FC contribution this scaling behavior is broken and we observe a shift of the critical frequency and a dropping of the corresponding maximum $L_{\max }\left(\omega_{\mathrm{cr}}\right)$ by increasing of the fluence. As example we show in the panel (A) of the Fig. $3 L_{\max }(\omega)$ for vanishing and unsaturated FC density using the complete Sellmeier equation at different fluencies.

iii) In the minimal depleted calculation (b) only the decreasing of $L_{\max }\left(\omega_{\mathrm{cr}}\right)$ as function of $F_{\mathrm{L}}$ occurs.

$i v)$ The optimum crystal length $l_{0}$ in the approach (a) using the linear approximation diverges for $\omega_{\mathrm{T}} \rightarrow \omega_{\mathrm{cr}}$, whereas the maximum od the generation length is finite and given by $L_{\max }\left(\omega_{\mathrm{cr}}\right)=2 / \alpha_{\mathrm{T}}\left(\omega_{\mathrm{cr}}\right)$.

v) Using the complete Sellmeier equation within the approach (a) as well using the depleted calculation (b) the optimum crystal length $l_{0}\left(\omega_{\mathrm{cr}}\right)$ becomes finite. Additionally, it becomes larger by increasing of the fluence.

The dependence of $l_{0}\left(\omega_{\mathrm{cr}}\right)$ on the fluence $F_{\mathrm{L}}$ is shown in the panel (B) of the Fig. 3 for the depleted calculation (b) as well as for different FC density within the approach (a). The comparison suggests that the linear approximation for the polarization in the depleted calculation (b) leads to an overestimation of the optimum crystal length. In general we note the importance of the details of the dynamics, i.e. the choice the FC density within the approach (a), for the determination of $l_{0}$.
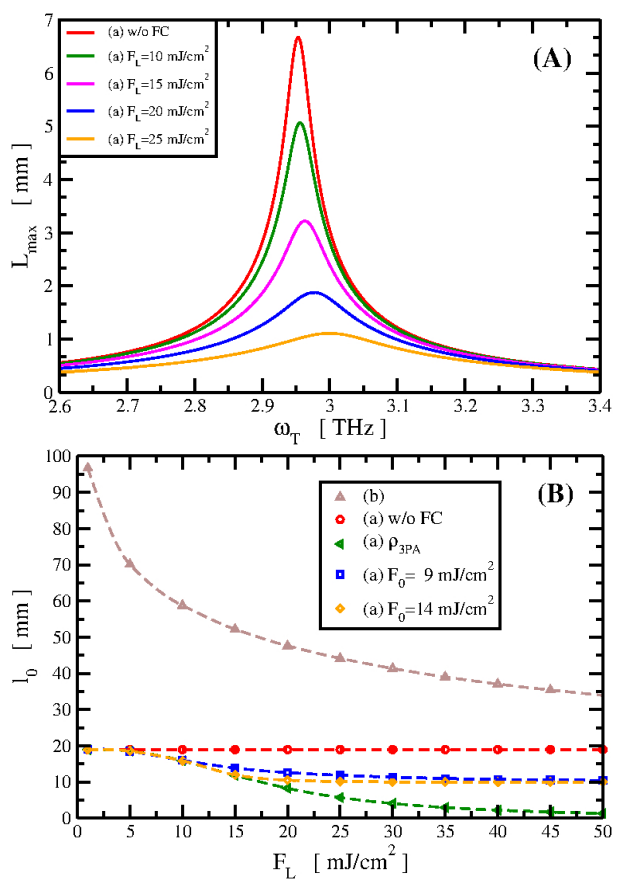

Figure 3: (A): $L_{\max }(\omega)$ for vanishing and for unsaturated FC density using the complete Sellmeier equation at different fluencies as indicated. (B): $l_{0}\left(\omega_{\mathrm{cr}}\right)$ as function of $F_{\mathrm{L}}$ for the depleted calculation (b) and different choices for the FC density within the approach (a) as indicated.

\section{CONCLUSION}

The influence of the optical properties of the lithium niobate crystal on the conversion efficiency as well as on the optimum crystal length of the generation of $\mathrm{THz}$ pulses has been investigated. We compare different approximation for the modeling of the generation dynamic (SVA vs. second order calculation) and of the influence of the free carries and we present a (minimal) depleted calculation.

We clearly show the importance of a consistent description of the optical properties as well as a detailed generation dynamics in order to develop the planned shot to shot feedback system, which have to perform the synchronization between electron bunch and the ultrashort laser with a time resolution of less than $1 \mathrm{fs}$.

\section{ACKNOWLEDGEMENT}

The work of S. Mattiello is supported by the German Federal Ministry of Education and Research (BMBF) under contract no. 05K16ROA. 


\section{REFERENCES}

[1] T. Tajima and J. M. A. Dawson, "Laser Electron Accelerator", Phys. Rev. Lett., vol. 43, no. 4, pp. 267-270, 1979.

[2] R. W. Assmann and J. Grebenyuk, "Accelerator Physics Challenges towards a Plasma Accelerator with Usable Beam Quality", in Proc. 5th Int. Particle Accelerator Conf. (IPAC'14), Dresden, Germany, Jun. 2014, pp. 961-964. doi : 10 . 18429/ JACOW-IPAC2014-TUOBBO 1

[3] S. Mattiello, A. Penirschke, and H. Schlarb, "Concept for the Minimization of the Electron Bunch Arrival-Time Jitter between Femtosecond Laser Pulses and Electron Bunches for Laser-Driven Plasma Wakefield Accelerators", in Proc. 6th Int. Beam Instrumentation Conf. (IBIC'17), Grand Rapids, MI, USA, Aug. 2017, pp. 157-160. doi : 10.18429/ JACOW-IBIC2017-TUPCC 55

[4] K. Ravi, D. Schimpf, and F. Kärtner, "Pulse sequences for efficient multi-cycle terahertz generation in periodically poled lithium niobate", Optics Express, vol. 24, no. 22, pp. 25582-25607, 2016.

[5] S. Mattiello, H. Schlarb and A. Penirschke, "Optical rectification for a new shot to shot feedback system for laser-driven plasma wakefield accelerators", in Proc. SPIE 10684, Nonlinear Optics and its Applications (SPIE:18), Strassbourg, France, May 2018, pp. 1068412, doi.org/10.1117/12. 2306429

[6] T. Hattori and K. Takeuchi, "Simulation study on cascaded terahertz pulse generation in electro-optic crystals", Optics Express, vol. 15, no. 13, pp. 8076-8093, 2007.

[7] A. Schneider, M. Neis, M. Stillhart, B. Ruiz, R. Khan, and P. Günter, "Generation of terahertz pulses through optical rectification in organic DAST crystals: theory and experiment", J. Opt. Soc. Am. B, vol. 23, no. 9, pp. 1822-1835, 2006.

[8] K. Vodopyanov, "Optical generation of narrow-band terahertz packets in periodically-inverted electro-optic crystals: conversion efficiency and optimal laser pulse format", Optics Express, vol. 14, no. 6, pp. 2263-2276, 2006.
[9] J .A. Fülöp et al., "Generation of sub-mJ terahertz pulses by optical rectification", Optics Letters, vol. 37, no. 4, pp. 557-559, 2012.

[10] S. Zhong et al., "Optimization of terahertz generation from $\mathrm{LiNbO} 3$ under intense laser excitation with the effect of three-photon absorption", Optics Express, vol. 23, no. 24, pp. 31313-31323, 2015.

[11] S. Mattiello, A. Penirschke, and H. Schlarb, "A Feedback System to Minimize the Electron Bunch Arrival-Time Jitter Between Femtosecond Laser Pulses and Electron Bunches for Laser-Driven Plasma Wakefield Accelerators", in Proc. 10th Int. Particle Accelerator Conf. (IPAC'19), Melbourne, Australia, May 2019, pp. 3843-3846. doi : 10. 18429/JACoW-IPAC2019-THPRBO20

[12] L. H. Deng et al., "Improvement to Sellmeier equation for periodically poled $\mathrm{LiNbO} 3$ crystal using mid-infrared difference-frequency generation", Optics Communications, vol. 268, no. 1, pp. 110-114, 2006.

[13] M. Schall, H. Helm, and S. R. Keiding, "Far Infrared Properties of Electro-Optic Crystals Measured by THz TimeDomain Spectroscopy", International Journal of Infrared and Millimeter Waves, vol. 20, no. 4, pp. 595-604, 1999.

[14] S. Mattiello, A. Penirschke, and H. Schlarb, "Optical Investigation to Minimize the Electron Bunch Arrival-time Jitter Between Femtosecond Laser Pulses and Electron Bunches for Laser-Driven Plasma Wakefield Accelerators", in Proc. 7th Int. Beam Instrumentation Conf. (IBIC'18), Shanghai, China, Sep. 2018, pp. 332-337. doi : 10.18429/ JACOW-IBIC2018-WEOAO2

[15] S. Mattiello, H. Schlarb and A. Penirschke, "Optical investigation to minimize the electron bunch arrival-time jitter between femtosecond laser pulses and electron bunches for laser-driven plasma wakefield accelerators", in Proc. SPIE 10902, Nonlinear Frequency Generation and Conversion: Materials and Devices XVIII, San Francisco, CA, USA, Feb. 2019, pp. 1090216, doi.org/10.1117/12.2508537 